\title{
Mechanical Modelling of Wire Reinforced Metal Matrix Extrusion Profiles
}

\author{
M. Wedekind ${ }^{1, a}, \mathrm{H}$. Baier $^{1, b}$ \\ ${ }^{1}$ Institute for Lightweight Structures (LLB) - Technische Universität München \\ Boltzmannstraße 15, 85747 Garching, Germany \\ awedekind@llb.mw.tum.de, bbaier@llb.mw.tum.de
}

Keywords: Metal matrix composite, compound extrusion, homogenization, failure criteria, mechanical properties, aluminium, steel

\begin{abstract}
Compound extruded unidirectionally reinforced profiles are heterogeneously reinforced metal matrix composites. Profiles made from this material show a different mechanical behaviour than classical composite components. Homogenized material properties are required for an efficient design process. Within this paper an approach is shown to provide homogenized data for stiffness and strength for compound-extruded components. The usability of the Tsai-Hill failure criterion is investigated, and shown for cases with negligible residual stresses. Load cases including thermal stresses cannot be investigated by the TsaiHill failure criterion. Within the failure investigation a procedure is shown to include residual and thermal stresses.
\end{abstract}

\section{Introduction}

Extrusion profiles are used widely for frame structures, e.g. space frames in the automotive industry. Metal matrix composites (MMCs) have been researched and used for some decades. Most MMCs have the advantage of a high mechanical performance coming with the disadvantage of randomly orientated reinforcements or very high production costs. The production and utilization of heterogeneously reinforced metal matrix composites profiles (HRMMC-profiles) is investigated within the DFG collaborative research centre, TR-10. This new material provides the opportunity to bridge the gap between low cost and high performance with. Wire reinforced extrusion profiles are metal matrix composite profiles with a number of steel or other types of wire reinforcements. These are investigated in this paper according to their behaviour under mechanical loading. An investigation of material properties of compound extruded composites with different configurations is shown in [1]. Compared to classical metal

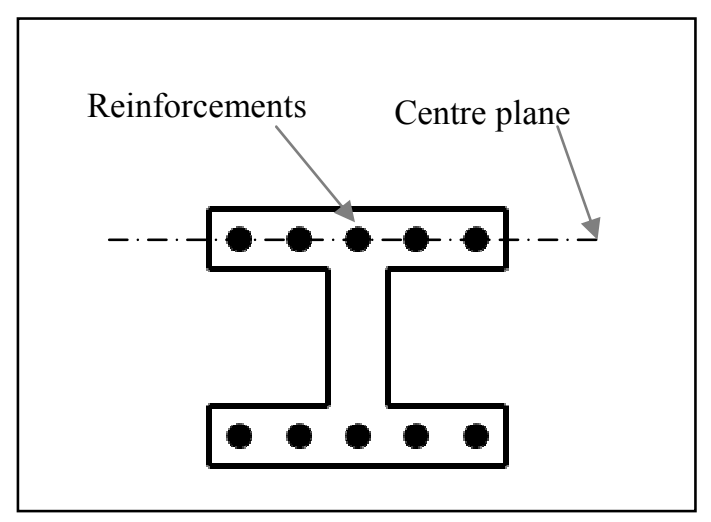

Figure 1: Cross section of a HRMMC profile matrix composites, the cross section of a single reinforcement is quite large. As a comparison, the diameter of a steel wire reinforcement is about $1 \mathrm{~mm}$ while the diameter of carbon fibres is about 5-10 $\mu \mathrm{m}$ [2] which allows a smooth distribution of the fibres. The reinforcements of the profiles investigated are located in the centre plane of the wall. The reinforcement volume fraction is quite low, similar volume fractions can be found in $\mathrm{SiC}$ fibre reinforced copper used for high temperature applications [3]. A scheme of a cross section of a HRMMC-profile is shown in Figure 1. Due to this specific setup, HRMMCs show a particular mechanical behaviour which is investigated in the following to provide information for design with profiles made from this material. 


\section{Analysis of existing metal matrix composites}

Metal matrix composites can be subclassified in particle reinforced composites, discontinuously fibre reinforced composites and continuously fibre reinforced composites. The properties of the different material types vary over a large range.

Particle reinforced composites include small particles with a size of about 5-20 $\mu \mathrm{m}$. This alters the mechanical properties and has an influence on the crack propagation under fatigue conditions [4].

Discontinuously fibre reinforced composites include fibres with a finite length. The load is introduced into the fibre by shear forces; therefore a critical length must be exceeded to utilize the strength of the fibre [5]. The load is carried partially by the reinforcements. Due to a short fibre length, the load is transferred between the fibres by the matrix. This causes a complex stress field within the matrix. Similar effects can be found at the boundaries and around the fibre damages within continuously reinforced composites.

The compound extruded profiles investigated are continuously reinforced components. These have an aluminium base material and steel wire reinforcements. In contrast to most continuously reinforced metal matrix composites, the reinforcements have a very large diameter. This results in a heterogeneous distribution of the stiffness at a macroscopic level.

Steel wire reinforced metal matrix composites have been investigated according to their mechanical properties [6] and crack propagation [7]. The influence of brittle intermetallic layers, generated by high temperature effects has been investigated [8], [9]. This material was used for the manufacturing of high pressure vessels with a service temperature up to $350^{\circ} \mathrm{C}$ [10].

During the compound extrusion process the reinforcements are embedded within the base material. The contact time between matrix material and the steel wires at a high temperature during the extrusion process is very short. Hence, there is no distinctive development of an intermetallic boundary layer [11].

\section{Homogenization of profile properties}

To enable an efficient design of structures made out of HRMMC-profiles and avoid large numerical models, the description of the material properties stiffness and strength should be provided with as little effort as possible. This description should enable the designer to realise a basic layout without being dependent on component tests within the early state of design.

An approach for a simplified calculation of a frame structure is shown. In the following the stiffness properties of the profiles are modelled by the role of mixture. The failure of the structure is determined by a failure criterion - either Tsai-Hill or a numerical criterion calculated by a FEM analysis.

For the undisturbed regions of the profiles a homogenization can be done easily. Due to the heterogeneous reinforcement boundary effects of the investigated profiles are very large. Boundary effects

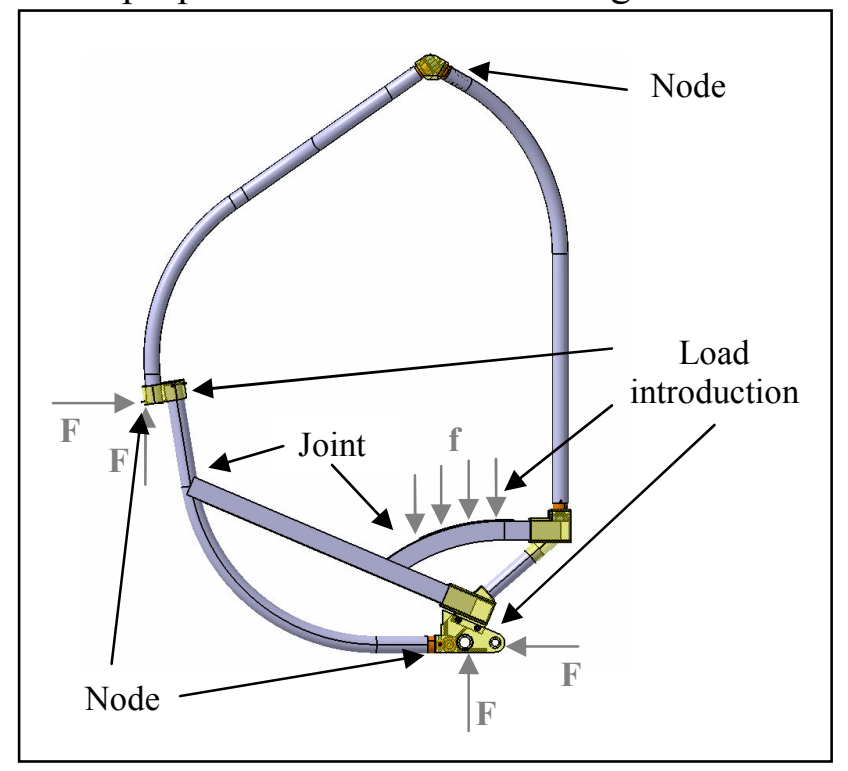

Figure 2: Framework of demonstrator can be found in areas with a high gradient of the stiffness, e.g. at load introductions and nodes of a framework. In this area the behaviour of the component are dominated by the design of nodes or load introduction, which results in a $3 \mathrm{D}$-stress state. A typical setup of a space frame 
the setup of the demonstrator is shown in Figure 2. The configuration is taken from a demonstrator scenario of the TR-10 project, which represents the space frame of a $\mathrm{C} 1$ motorcycle.

Joints and nodes require either a more detailed modelling of the profiles or a further investigation e.g. FEM analysis using substructuring. For a detailed FEM analysis, the loads on the nodes are taken from a global model with homogenised properties. Within the following an analysis of the behaviour of undisturbed profiles is shown. Further investigation will be done on the profile homogenization in complex regions of nodes, joints and loading points.

Because the design loads cause only a linear elastic deformation, the homogenization of the elastic region is important. The homogenization of material properties for classical continuous fibre reinforced composites is well known. There are different methods to estimate the stiffness parallel and perpendicular to the fibres. An overview is shown in [5].

The predominant loads of the profiles are stresses in extrusion direction, bending forces and shear loads due to torsional moments. For elastic strain the stiffness of the profile in fibre direction can be described by the well known rule of mixture (ROM), see Equation (1). $E$ is Young's modulus and $\varphi$ is the fibre volume fraction.

Due to a homogeneous strain field in this case, there is no influence of the stiffness's heterogeneous distribution on the material properties.

$$
E_{\text {compound }}=E_{\text {fibre }} \cdot \varphi+E_{\text {matrix }} \cdot(1-\varphi)
$$

To calculate the bending stiffness, the distribution of the strain within the profile has to be taken into account. In this case the effect of the reinforcement wires is dominated by their locations. These are displayed by the configuration's moment of inertia of all reinforcements $I_{\text {fibres }}$.

$$
I_{\text {compound }}=\frac{E_{\text {fibre }} \cdot I_{\text {fibres }}+E_{\text {matrix }} \cdot I_{\text {matrix }}}{E_{\text {compound }}}
$$

With this information the basic stiffness properties for flexural loads and loads in fibre direction are provided. The stiffness properties in perpendicular direction are dependent on the reinforcement position, and therefore no analytical investigation was performed.

\section{Failure mechanism and failure criteria}

For a failure analysis, 'failure' must be defined. Metal matrix composites with plastic matrix show different failure modes. For loading in the reinforced direction, the first failure mode occurs when the matrix's yield limit is exceeded. The second failure mode is a plastic deformation of the matrix exceeding a certain value, which leads to plastic deformation of the component [13]. In [13] this behaviour is utilized to take use of the very high ultimate stresses of this material. The application of the different failure definitions is dependent on the purpose. A further failure mode is known for steel wire reinforced metal matrix composites with a brittle intermetallic layer. Cracking of this layer causes notches and leads to a premature failure of the reinforcements [9]. This layer occurs due to diffusion at high temperatures. The maximal diffusion length during the extrusion process investigated is about $250 \mathrm{~nm}$. Therefore only a very thin boundary layer is created [11], which is assumed to be not critical because of the non-brittle fibres. For loading in direction perpendicular to the fibres additional failure modes can occur, e.g. debonding of the reinforcements. Since the bonding 
between base material and reinforcement is very strong, a perfect bonding is assumed in the following investigation. This is shown in [11].

For most design cases exceeding the yield limit of the matrix, failure can be assumed. Beyond this point a plastic deformation of the component remains and residual stresses are generated. Furthermore, due to work hardening the material properties may be dependent on the load history.

Within a compound extruded component, large areas of undisturbed material exist. Hence it can be assumed that the reinforcing has no strengthening effect on the matrix properties. To achieve a strengthening effect these areas must be very small (in the order of $10 \mu \mathrm{m}$ ) [15].

\section{Failure of uniaxial loaded HRMMCs}

Axial loads refer to loads in fibre direction in the following. Loads perpendicular to the fibre direction will be called perpendicular loads. The associated strength will be called perpendicular strength. For axial loading, strain in matrix and reinforcements are equal. For equal Poisson's ratios of matrix and reinforcement following relations, given in (3), are valid. Since the Poisson's ratios of steel and aluminium are similar, the error is small. ' $\sigma_{\text {fibre }}$ ' and ' $\sigma_{\text {matrix }}$ ' describe the stresses in axial direction in the reinforcements and the matrix. ' $\sigma_{\text {compound } \|}$ ' describes the averaged stress of the component in axial direction.

$$
\frac{\sigma_{\text {fibre }}}{\sigma_{\text {compound } \|}}=\frac{E_{\text {fibre }}}{E_{\text {compound } \|}} \quad \frac{\sigma_{\text {matrix }}}{\sigma_{\text {compound } \|}}=\frac{E_{\text {matrix }}}{E_{\text {compound } \|}}
$$

With this information the yield stress of the compound can be calculated. In Equation (4) the linear correlation between the reinforcement fraction and the maximal linear elastic stress of the component is shown.

$$
\sigma_{\text {max } \_ \text {compond } \|_{\|}}=\sigma_{\text {max }_{-} \text {matrix }} \cdot\left[1-\left(1-\frac{E_{\text {fibre }}}{E_{\text {matrix }}}\right) \cdot \varphi\right]
$$

2D-stress fields resulting from loads perpendicular to the reinforcement direction are more complex. These are dominated by effects of the heterogeneous stiffness.

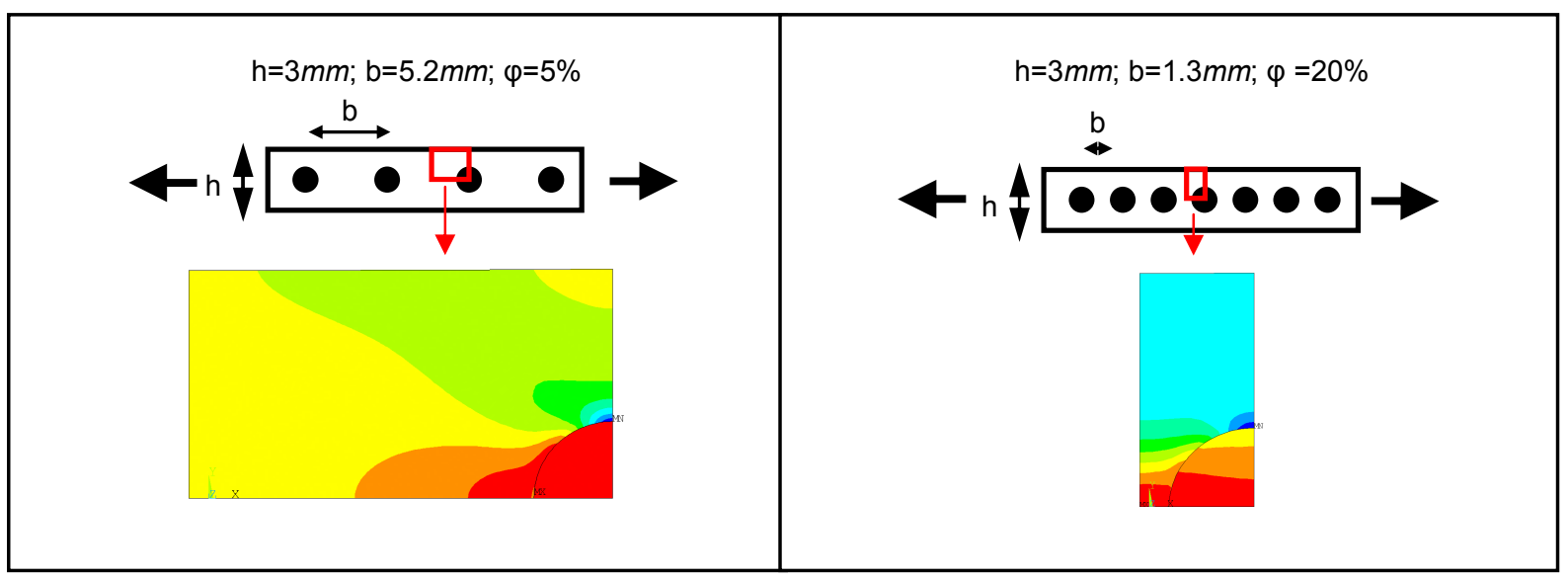

Figure 3: Stress field perpendicularly loaded profile 
In Figure 3 the schematic stress fields of perpendicularly loaded profile cut outs are shown. These stress fields are dependent on the height $h$ of the profile and the distance $b$ between the reinforcements. All reinforcements are located in the centre layer. This leads to stiffening and stress concentration within this layer, dependent on the distance between the reinforcement elements. The relation between the reinforcement fraction and the perpendicular strength is plotted in Figure 4. The assumed material properties are shown in Table 1. ' $\sigma_{\text {compound }}$ ' is the maximal average perpendicular stress on the component without plastic deformation within the matrix.

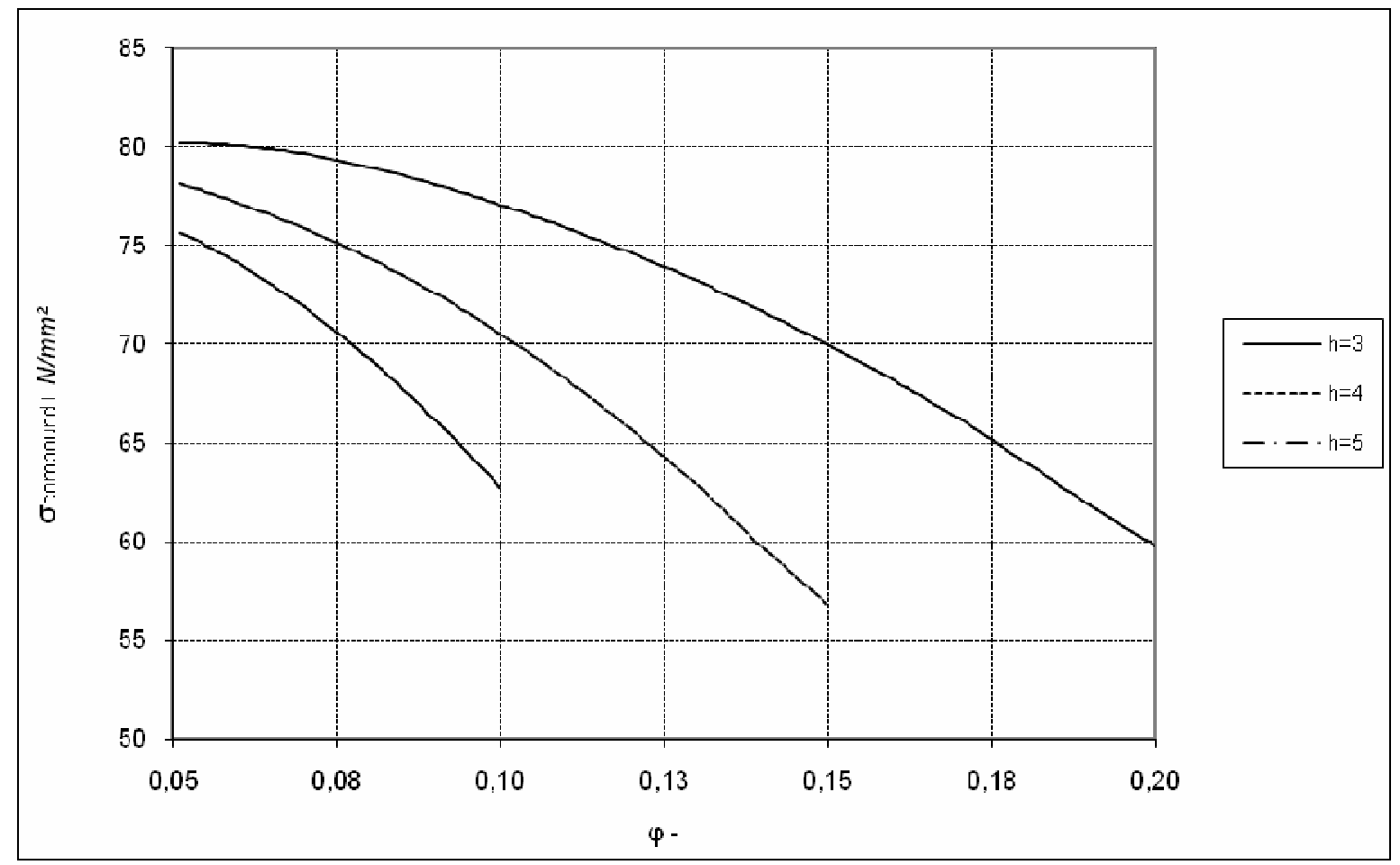

Figure 4: Perpendicular strength as function of reinforcement fraction $(\varphi)$

\section{Failure of steel wire reinforced profiles under multi axial loading}

To analyse the multi axial failure for steel wire reinforced aluminium, a FEM analysis is performed. A cut out of the component is modelled and investigated. The failure loads for this model are calculated with the same assumptions as discussed. Exceeding the yield stress of

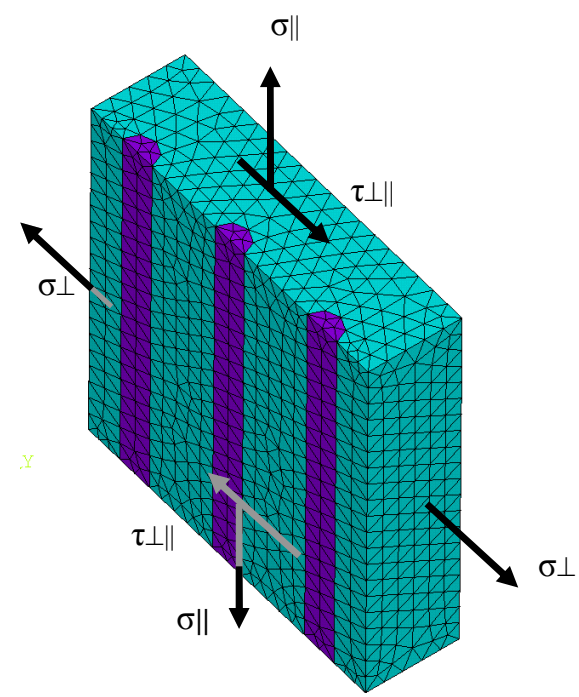

Fig. 5: FE-Model for stress analvsis the matrix is assumed as failure. To eliminate boundary effects within the model, a specimen with three reinforcements is modelled. Only the data for the inner area is used for evaluation. Because of the shear stress state only one symmetry can be assumed.

The model has linear material properties for fibre and matrix. The connection between fibre and matrix is assumed as perfect bonding realised by merging the nodes. The material properties are shown in Table 1. The diameter of the reinforcements is $1 \mathrm{~mm}$.

With this model the stress fields for the basic load cases (axial load, perpendicular load and shear load) are calculated. The data of the resulting stress fields is stored for further processing. 
Since the boundary of linear behaviour is of interest, the various load cases can be calculated by linear superposition of the basic load cases. For each load case a load vector is defined, which consists of a relation between $\sigma \|$ (axial direction), $\sigma \perp$ (perpendicular direction) and $\tau \perp \|$ (shear). The field of equivalent stresses is calculated for this load combination with the von Mises hypothesis. The load vector is scaled to a size that the yield stress of the matrix is met. This is calculated for a defined set of load vectors and the result is shown as a 'yield surface'. The gained data is compared with the Tsai-Hill failure criterion to evaluate its usability. The objective of using such a failure criterion is the simplicity of its application. For an analysis with a Tsai-Hill failure criterion only a small amount of data is required. The Tsai-Hill failure criterion is an 'anisotropic von Mises' criterion. It was developed by Hill for the material description of anisotropic, rolled sheet metal. Afterwards Tsai adapted it for use with unidirectional laminates [16]. Tsai-Hill is a wide spread failure criterion for the analysis of

Material properties for FEM-model

\begin{tabular}{|l|r|c|}
\hline Young's modulus matrix & 70000 & $\mathrm{MPa}$ \\
\hline Young's modulus fibre & 210000 & $\mathrm{MPa}$ \\
\hline Poison ratio matrix & 0.33 & - \\
\hline Poison ratio fibre & 0.29 & - \\
\hline a-matrix & 0 & $1 / \mathrm{K}$ \\
\hline a-Faser & 0 & $1 / \mathrm{K}$ \\
\hline Yield stress matrix & 100 & $\mathrm{MPa}$ \\
\hline
\end{tabular}

Table 1: Material properties unidirectional laminates.

The information needed for Tsai-Hill, the failure loads for $\sigma_{\| \max }, \sigma_{\perp \max }$ and $\tau_{\perp \| \max }$, are taken from the yield stresses calculated by the FEM analysis. At these points the deviation between the FEM analysis and the Tsai-Hill criterion is zero, as defined. The definition of the Tsai-Hill failure criterion is shown in Equation (5).

$$
\left(\left(\frac{\sigma_{\|}}{\sigma_{\| \max }}\right)^{2}+\left(\frac{\sigma_{\perp}}{\sigma_{\perp \max }}\right)^{2}-\frac{\sigma_{\|} \cdot \sigma_{\perp}}{\sigma_{\| \max }{ }^{2}}+\left(\frac{\tau_{\perp \|}}{\tau_{\perp \| \max }}\right)^{2}\right)^{0.5} \leq 1
$$

With a comparison of Tsai-Hill and the FEM-failure analysis, the usability of the global failure criterion is investigated. The result of the comparison is shown graphically as a deformed sphere. The vector from the origin to a point at the surface describes the load combination. Its length is defined as the strength of the FEM failure analysis divided by the yield stress calculated with Tsai-Hill. A perfect sphere with the radius 1 represents exact correlation between both criteria. The colouring shows the deviation between the FEM failure and the yield stress calculated by Tsai-Hill. In Figure 6 the procedure is explained. 


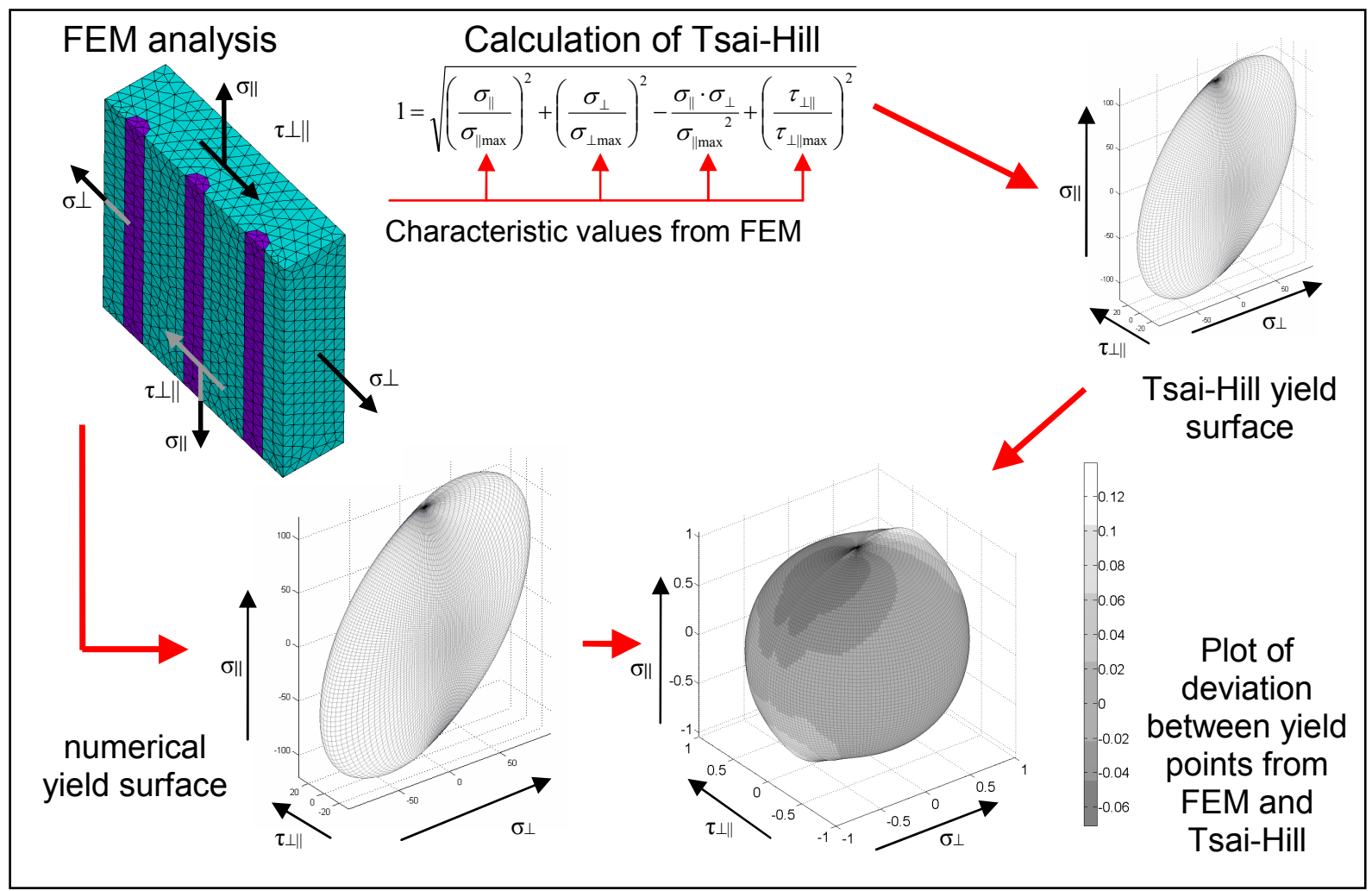

Figure 6: Procedure for comparison Tsai-Hill failure criterion with numerical failure analysis

\section{Results}

In the following a comparison of the analysis of different configurations of steel wire reinforced aluminium is shown. For the setup there are three design variables: the diameter of the reinforcement elements, the thickness of the material and the reinforcement fraction. Within this investigation only reinforcements with a diameter of $1 \mathrm{~mm}$ are taken into account. To enable the comparison of configurations with different reinforcement volume fractions a thickness of $3 \mathrm{~mm}$ is chosen. Since the reinforcements are only located in the inner area of the composite, the maximal possible reinforcement volume fraction is limited by the height of the component. With a thickness of $3 \mathrm{~mm}$ a reinforcement fraction up to $20 \%$ is possible without contact between the reinforcements - disregarding constraints of production.

The following figures show the yield surface calculated by FEM on the left. On the right the plots of the deviation between the FEM and the analytical solution is pictured out by the colouring. Their ratio is shown by the distance from the origin. In Figure 7 the configuration with a low reinforcement volume fraction of 5\% is shown. The maximal deviation between the FEM solution and the Tsai-Hill failure criterion is approximately $8 \%$. For the load cases with only axial or perpendicular or shear loading the deviation is, according to the definition, exactly zero. The highest deviation is found in the area with approximately equal loading in axial and normal directions with small shear load.

In Figure 8 the results of a high reinforcement volume fraction of $20 \%$ is shown. The deviation between FEM and Tsai-Hill is increased for the larger volume fraction. The maximal deviation occurs in the case of approximately equal loads on both axes without shear loading. The highest deviation is approximately $12 \%$. 


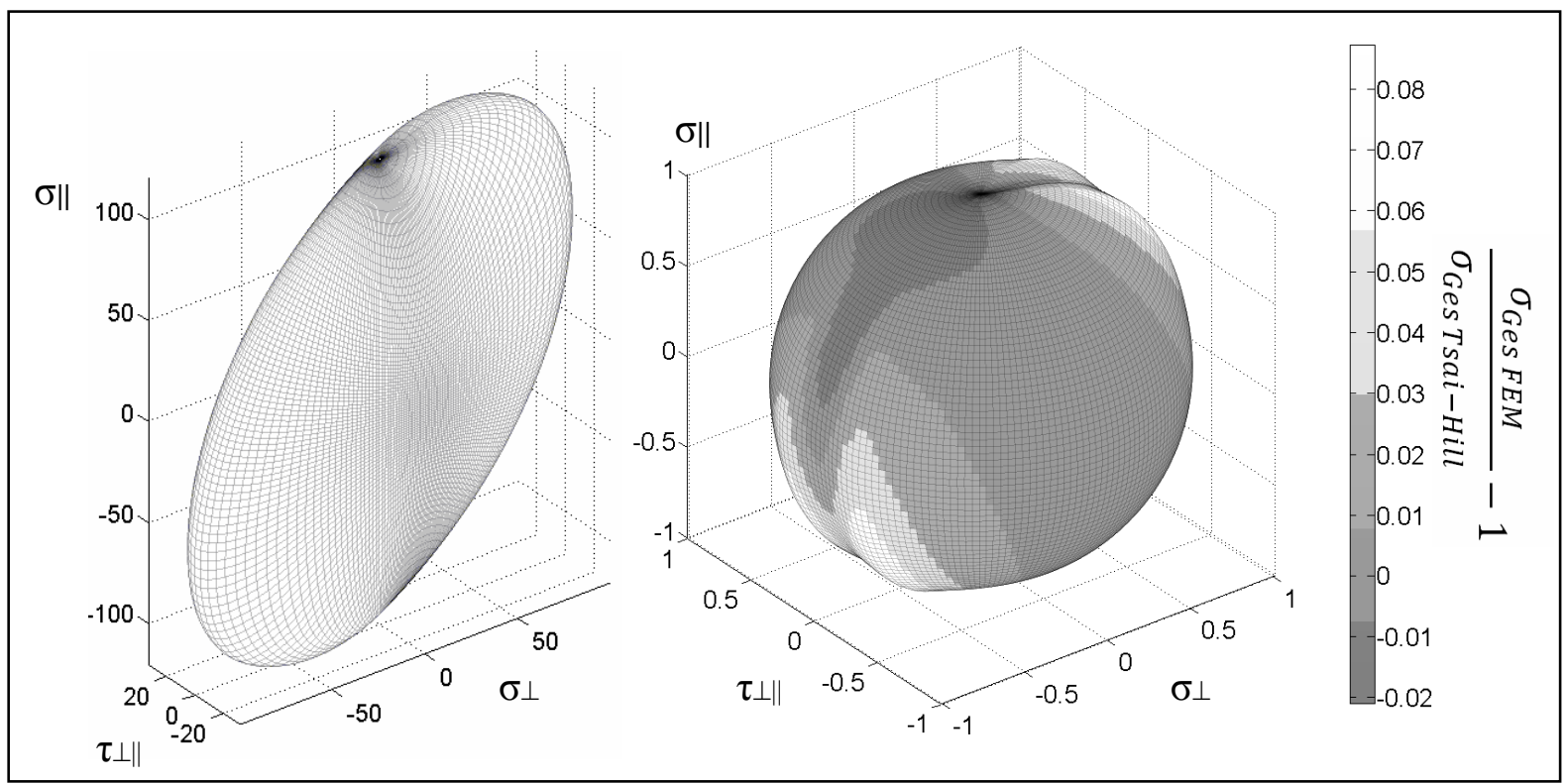

Figure 7: Failure surface (left) and deviation from Tsai-Hill with reinforcement fraction 5\% (right)

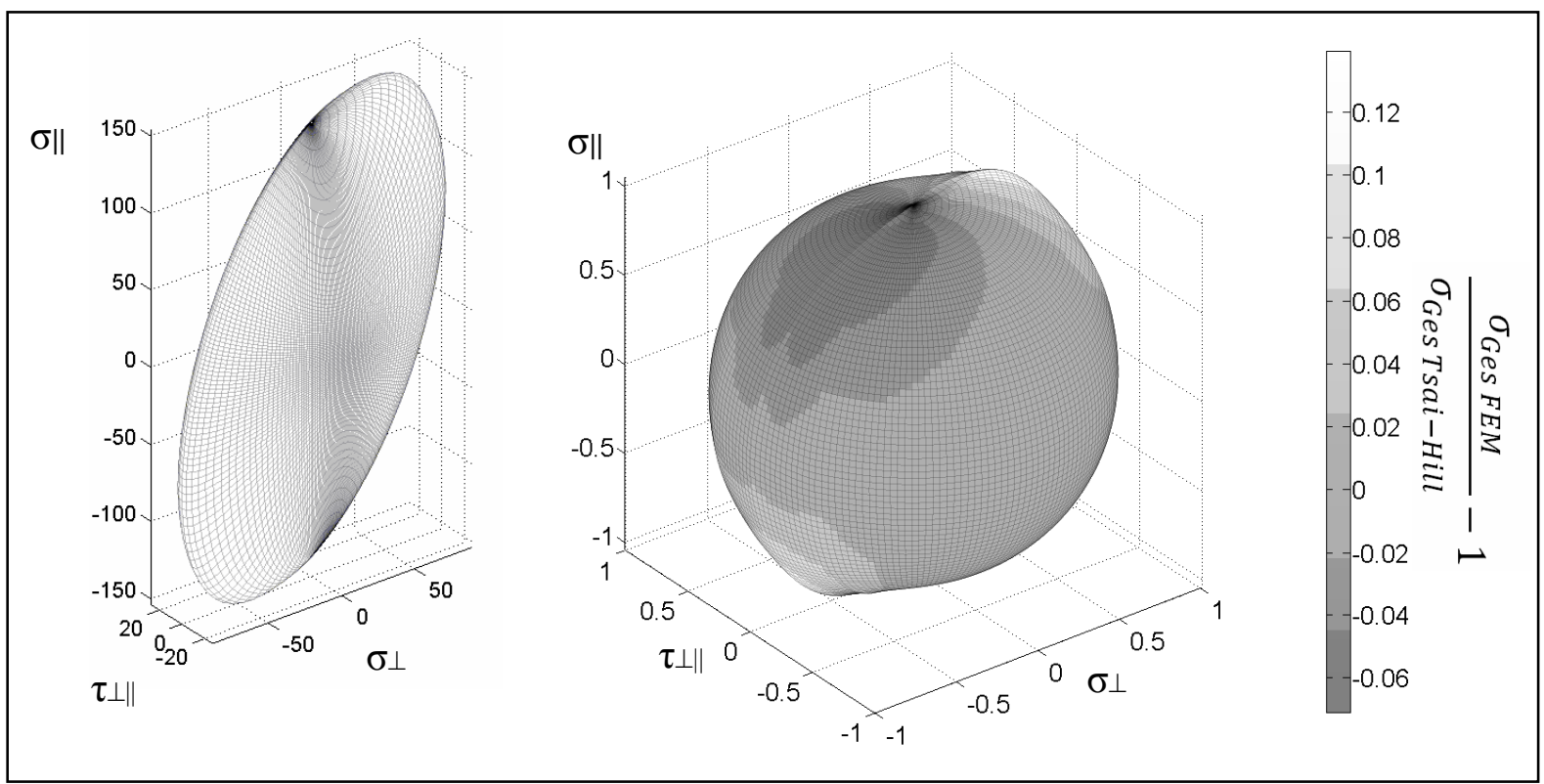

Figure 8: Failure surface (left) and deviation from Tsai-Hill with reinforcement fraction 20\% (right)

\section{Discussion of failure criterion}

In case of pure axial loading, the whole cross section of the matrix reaches the yield limit simultaneously. This leads to a plastic deformation of the entire matrix material as well as the component. After decreasing the load residuals stresses are left.

In case of a perpendicular loading the complex stress field is dominated by effects of the stiff steel wire in the weak matrix. By definition, failure of the profile occurs with the first plastic deformation of the matrix material. In case of perpendicular loading only a small area of the matrix is affected. The influence of this plastic deformation on the component's global deformation is small. The stress fields are altered and the material properties are changed due to strain hardening. This has a significant impact on the fatigue properties and is assumed as a failure. 
The application of this failure mode is useful for the layout of a framework for limit loads. In this case no plastic deformations are allowed. For a layout of an overload which exceeds the yield limit this approach is not valid.

\section{Thermal effects on mechanical behaviour}

For a typical framework the coefficient of thermal expansion (CTE) in axial direction is especially important. A CTE mismatch within a structure leads to thermal stresses and deformations.

The compound consists of two materials with a different coefficient of thermal expansion. This leads to residual stresses when exposed to a temperature load. The profile is produced at a temperature of approximately $550^{\circ} \mathrm{C}$ [14]. Thermal residual stresses result from the cool down process.. These depend on the recrystallisation temperature of the matrix material. The exact effect of the production on the residual stress fields will be investigated in further research. Residual stresses have been investigated by the subproject A3 of TR-10 [15]. In the following the general effect of a temperature offset on the component is investigated.

For a continuous fibre reinforced composite the coefficient of thermal expansion in fibre direction can be calculated by the internal balance of the forces. This leads to Equation (6) [5]. The coefficient of thermal expansion is represented by $\alpha$.

$$
\alpha_{\text {compound } \|}=\frac{E_{\text {fibre }} \cdot \alpha_{\text {fibre }} \cdot \varphi+E_{\text {matrix }} \cdot \alpha_{\text {matrix }} \cdot(1-\varphi)}{E_{\text {fibre }} \cdot \varphi+E_{\text {matrix }} \cdot(1-\varphi)}
$$

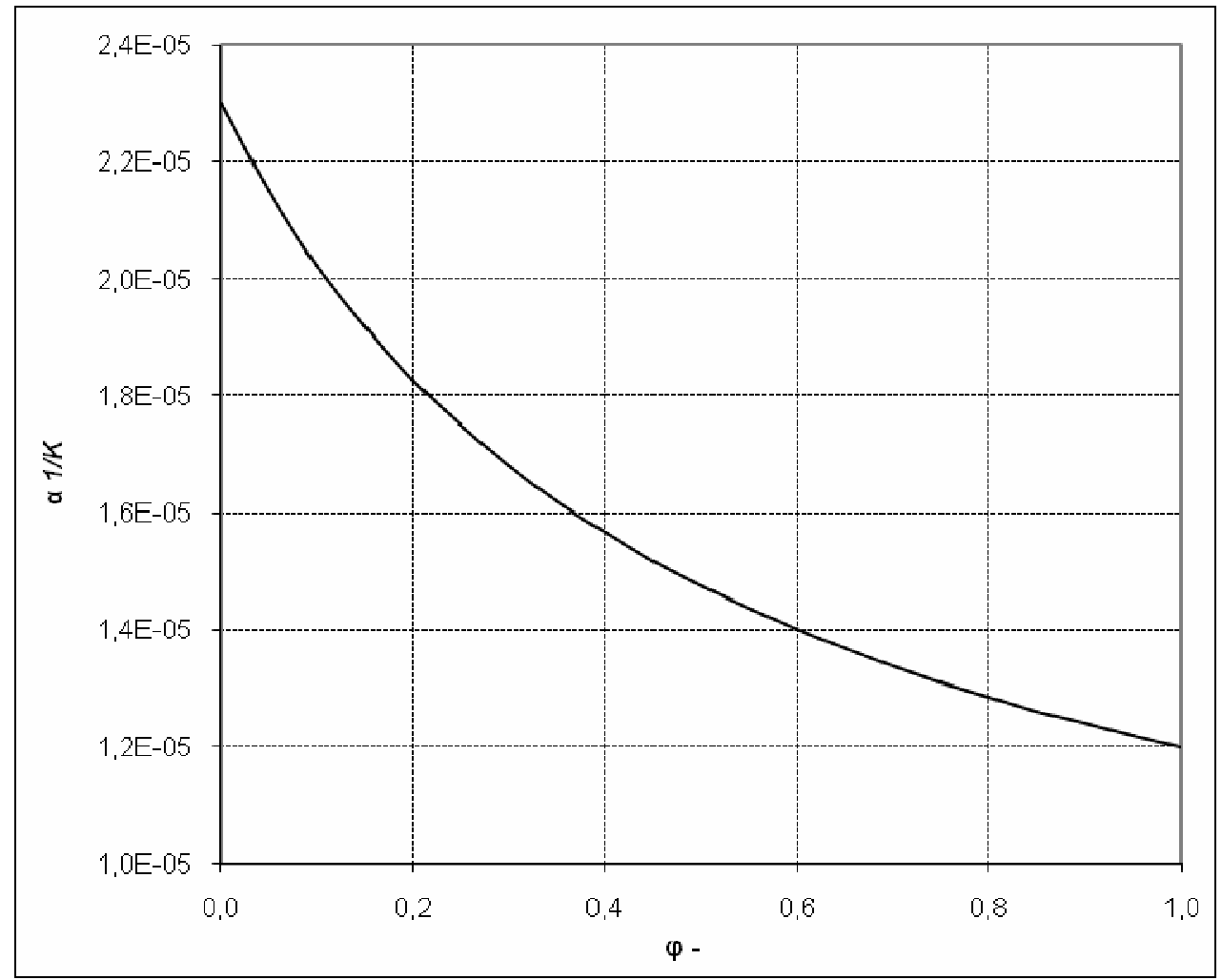

Figure 9: Coefficient of thermal expansion $(\alpha)$ as function of reinforcement volume fraction $(\varphi)$

The CTE of the material can be adapted within a certain range. For the materials used currently the CTE can be chosen in between the properties of aluminium and steel. The 
relation between CTE and reinforcement volume fraction is shown in Figure 9. Usage of other reinforcement materials (e.g. carbon fibres) will expand this range.

\section{Thermal and residual stresses}

The mismatch of CTE in the hybrid component causes thermal stresses. The thermal stress fields are multi-axial and depend on the geometry distribution of the fibres. Within the heterogeneous compound, the location of the reinforcement has a dominating influence.

These thermal stresses cause a change of the mechanical behaviour of the component. For the following investigation a temperature drop from a stress free state is assumed. A CTE of the matrix much higher than the CTE of the reinforcements results in a tensile stress within the matrix and a compressive stress within the fibres. The yield loads of the component are transferred to the compressive region. During the production process the profile passes a large temperature drop. The influence of this temperature drop and relaxation effects of matrix material will be investigated in further research.

With the FEM failure analysis an integration of thermal and residual stresses was performed, similar as described above. For the calculation the stress field is combined from variable part (external loads) and an invariant part (thermal and residual stresses). To calculate the yield limit an iteration process was implemented, which approaches the yield boundary from both sides and creates an interval containing the yield limit. This interval is reduced to a small size, and therefore convergence of the iteration process can be assumed.

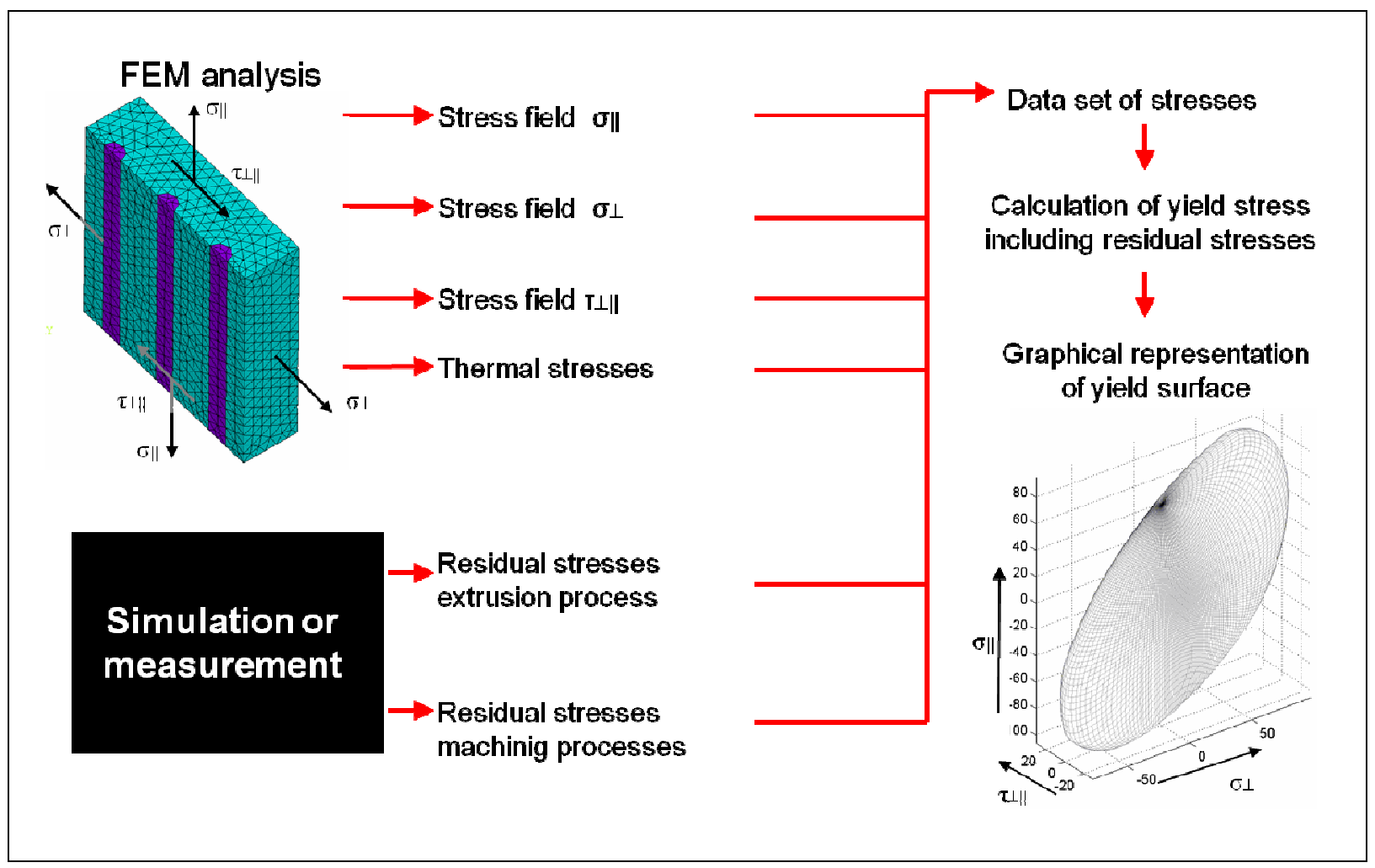

Figure 10: Scheme calculating yield load with residual stresses

In Figure 10 the scheme of the calculation of the yield surface, including thermal and residual stresses, is shown for the configuration with a reinforcement volume fraction of $5 \%$ and thickness of wall of $3 \mathrm{~mm}$. 


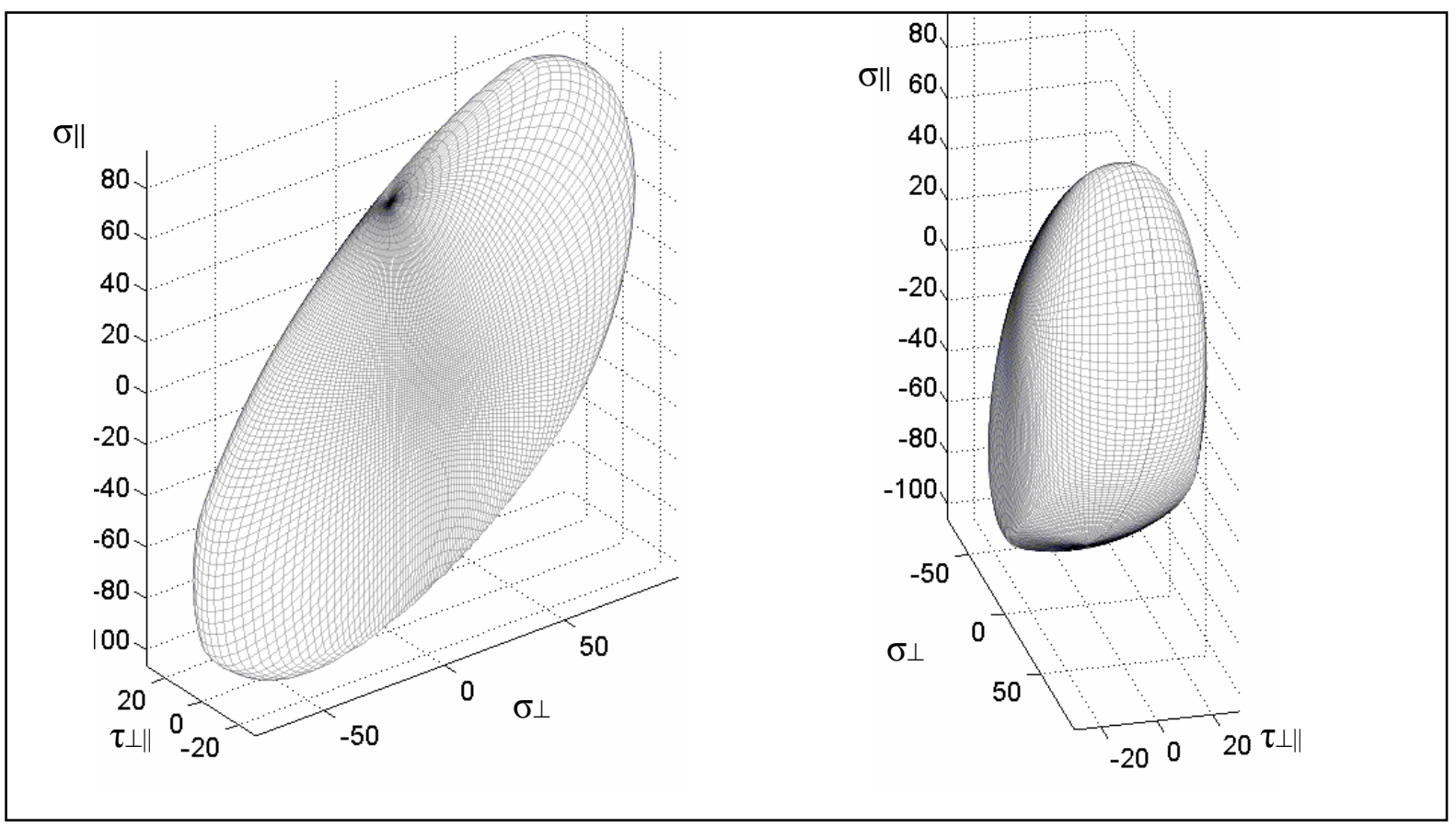

Figure 11: Yield surface with thermal offset $\Delta \mathrm{T}=-50^{\circ} \mathrm{C}$ from stress free state

In Figure 11 the yield surface for an aluminium matrix profile with a temperature offset of $-50^{\circ} \mathrm{C}$ from a stress free state is shown. The yield stresses are moved to the compressive area. The tensile yield strength in axial direction is reduced from $110 \mathrm{MPa}$, without thermal loads, to about $60 \mathrm{MPa}$. Another interesting effect is the cut off in the compressive area. Due to the complex stress fields around the reinforcements not only an offset of the yield surface occurs, but also a change of the shape. The yield surface is no ellipsoid any more. Within the compressive region a flattened area is found, shown on the left in Figure 11. For an analysis of a component with thermal or residual stresses, evaluating a component with a failure criterion like Tsai-Hill is not possible.

\section{Summary}

The homogenized material properties of extrusion profiles have been investigated with the objective to provide an easy to use failure criterion for design of such components. A comparison of the Tsai-Hill failure criterion with a numerical calculation was performed. For simple cases without residual stresses, the deviation between Tsai-Hill and the numerical analysis is small. The influence of a stress field, resulting from a temperature, on the yield behaviour on a component was analysed. Under thermal loads an analysis with a global failure criterion is not possible. In further investigations the influence of the manufacturing process and plastic deformations will be integrated. The failure criterions will be compared and verified with results from biaxial testing. This paper is based on investigations of the collaborative research centre SFB/TR 10 which is kindly supported by the German Research Foundation (DFG).

\section{Acknowledgment}

This paper derives from investigations within the scope of the Collaborative Research Center/TR10, which is kindly supported by the German Research Foundation (DFG). 


\section{References}

[1] K. A. Weidenmann, E. Kerscher, V. Schulze, D. Löhe: Mechanical properties of compound-extruded aluminium-matrix profiles under quasi-static loading conditions. Advanced Materials Research Vol. 10, pp. 23-34,2006

[2] H. Schuermann: Konstruieren mit Faser-Kunststoff-Verbunden. Springer, Heidelberg, 2005

[3] C. R. Popescu: Processing and Characterisation of SiC-Fibre Reinforced Cu-Matrix Composites, http://nbn-resolving.de/urn/resolver.pl?urn:nbn:de:bvb:91diss2004031818668

[4] M.T. Milan and P. Bowen: Fatigue Crack Growth Resistance of SiCp Reinforced Al Alloys: Effects of Particle Size, Particle Volume Fraction, and Matrix Strength. Journal of Materials Engineering and Performance, Volume 13 No. 5, pp. 612-618, 2004

[5] A. Zeidler: Abschätzung der Versagensgrenzen von Faserverbundwerkstoffen mit duktiler Matrix. Fortschr.-Ber. VDI Reihe 18 Nr. 236. Düsseldorf: VDI Verlag 1998

[6] M. Kh. Shorshorov, L. N. Moguchii, L. M. Ustinov, V. I. Zhamnova and M. P. Shebatinov: Investigation of the failure in tension of an aluminum alloy reinforced with steel wire. Strength of Materials, Volume 7, No. 8, pp. 961-965, 1975

[7] M. Kh. Shorshorov, V. G. Kudryashov, L. M. Ustinov, V. I. Zhamnova and E. N. Rudnitskii: Resistance to crack propagation in an aluminum-alloy-steel-wire composite. Strength of Materials, Volume 10, No. 4, pp. 378-381, 1978

[8] M. K Rybal'chenko and L. M. Ustinov: Effect of the fiber-matrix interface on the plasticity and strength of fibrous composites. Strength of Mat., Volume 4, No. 9, 1972

[9] M. Kh. Shorshorov, L. M. Ustinov, A. M. Zirlin, V. I. Olefirenko and L. V. Vinogradov: Brittle interface layers and the tensile strength of metal matrix-fibre composites. Journal of Materials Science, Volume 14, No 8, pp. 1850-1861, 1979

[10] E. R. Khismatulin, R. S. Yusupov, S. I. Kopylov and A. P. Alyab'ev: High-pressure vessels of aluminum-steel-wire composite material. Chemical and Petroleum Engineering, Volume 23, No 5, pp. 213-215 1987

[11] K. A. Weidenmann, Eberhard Kerscher, Volker Schulze, Detlef Löhe: Werkstoffsysteme für verstärkte Leichtbauprofile. VDI Fortschrittsberichte, Nr. 661, pp. 69 -105, 2007

[12] K. A. Weidenmann, E. Kerscher, V. Schulze, D. Löhe: Mechanical Properties of Compound-Extruded Aluminium-Matrix Profiles under Quasi-Static Loading Conditions. Advanced Materials and Research, Volume 10, pp. 23-33, 2006

[13] A. J. Svobodnik,: Numerical Treatment of the Elastic-Plastic Macromechanical Behaviour of Longfiber-Reinforced Metal Matrix Composites. Fortschr.-Ber. VDI-Reihe 18, Nr. 90, 1990

[14] A. Klaus, D. Becker, M. Kleiner: Three Dimensional Curved Profile Extrusion- First Results on the Influence on Gravity. Advanced Material Research, Volume 10, pp. 5-10, 2006

[15] K. A. Weidenmann: Werkstoffsysteme für verbundstranggepresste Aluminiummatrixverbunde. Schriftenreihe Werkstoffwissenschaft und Werkstofftechnik, Vol. 33, 2006

[16] A. Kelly, G. J. Davies: The Principles of the Fibre Reinforcements of Metals. Metal. Rev. Vol. 10, 1965

[17] A. K. Kaw: Mechanics of Composite Materials. CRC Press LLC, 1997 